\title{
INNERVATION OF THE HEART OF LIGIA OCEANICA
}

\author{
By J. S. Alexandrowicz \\ From the Plymouth Laboratory
}

(Plate I and Text-figs. I-3)

During my stay at the Plymouth Laboratory in I93I I had an opportunity of investigating the innervation of the heart of Ligia oceanica, and of making some experiments on the function of the ganglionic trunk which constitutes the main element of its innervation. In a short note describing these experiments (Alexandrowicz, I93I) it was stated that a description of the arrangement of the nerve elements would appear in a short time. However, on later examination of the preparations it seemed to be desirable to verify some of the findings, but the same animal was no longer available. Another isopod, Mesidotea entomon, with which I tried to continue the work during my visit to the Marine Biological Laboratory in Hel, proved to be much less suitable for this kind of investigation despite its larger size. Later events, unfavourable for scientific work, caused the loss of all my preparations and a further delay. It is only now, thanks to the chance of working again at the Plymouth Laboratory, that I have been able to resume my investigations.

I wish to record my gratitude to Mr F. S. Russell, F.R.S., for his kind help in preparing the manuscript.

\section{MethoD}

The observations recorded below were made on preparations stained with methylene blue, either by injection or by immersion of the tissues, or by a combination of the two methods (for details see Alexandrowicz, I932, I95I). None of the methods or the various modifications, such as changing the concentration of the solutions, the temperature, or the $\mathrm{pH}$ have given fully satisfactory results since certain nerve elements, e.g. the ganglion cells in the main trunk, stain but rarely, while others, such as the nerves running alongside the aorta, stain fairly well in the part of their course near the heart but not at all at their origin from the ganglia in the anterior region of the animal. The latter phenomenon may be partly due to the effect of the secretion of the digestive glands which hinders the staining. It is therefore advisable when dissecting the animals to remove these glands as quickly as possible and to wash the tissues thoroughly with sea water. It is, however, difficult to prevent the organs in the anterior region of the animal from being contaminated by the secretion of the glands.

The heart can be exposed either from the ventral or from the dorsal side. 
The first way is much the easier. It is sufficient to cut out the dorsal body wall and fix it to a paraffin plate with the ventral side turned upwards: the heart then appears in view partly covered by gonads which should be removed. In order to expose the nerve elements situated on the inner surface of the heart tube the ventral wall of the latter must be cut through along its median line. This should be done under a dissecting microscope with fine scissors, and it is not as difficult as it might appear owing to the fact that the walls of the heart are attached to the surrounding organs in such a way that they do not collapse. It is also very convenient both for staining and for observation that the ventral wall of the heart remains wide open after it is split, leaving the inside of the heart clearly visible. In Mesidotea entomon the heart tube is flaccid and it is much more difficult to split it and observe the inside.

A serious inconvenience to observation of the heart nerves in Ligia is due to chromatophores containing a pigment which does not dissolve in alcohol, so that the pigmented terga must be removed. This is the most difficult manipulation in the whole later procedure. It can be done best after the fixation when the preparations are being washed in water. The pigmented cells may also be present in the pericardial diaphragm and it is not possible to remove them at this spot. It is therefore preferable to choose animals which are less pigmented.

Access to the heart from the dorsal side is much more difficult, since it is necessary to cut out the terga one after another and to remove the dorsal muscles covering the heart. This is a tiresome operation, but this method exposes the dorsal surface of the heart and gives a view of its nerves from en additional aspect.

\section{OBSERVATIONS}

\section{On the Anatomy of the Heart of Ligia oceanica}

The heart of $L$. oceanica is shaped like a tube which in the largest specimens is about $15 \mathrm{~mm}$. long. It is composed of a single layer of muscle fibres turning in right-handed spirals and of a thin coat of connective tissue (Text-fig. I A; Pl. I, figs. I, 3). On the inner side of the muscle layer there is a plexus of strands of connective tissue elements composed mostly of cells with highly refringent inclusions, presumably of fatty material (Pl. I, fig. 2).

The heart wall is pierced by four ostia situated alternately on either side as shown in Text-fig. I B. Delage (I88I) and Gordon Hewitt (1907) state that there are two only, but I have always found four of them, and they appear so distinctly in preparations in which the heart wall has been stretched (Pl. I, fig. 3) that there can be no doubt on this point. It may be mentioned that on the muscle fibres bordering the ostial orifices some cells can be observed staining deeply with methylene blue. These are mesenchymal elements differing in appearance from those arranged in the plexiform strands on the inside of the heart. 
Nine arteries arise from the heart. One called the aorta runs forward in the mid-line, the others are four paired arteries situated in the anterior half of the heart as shown in Text-fig. I A.

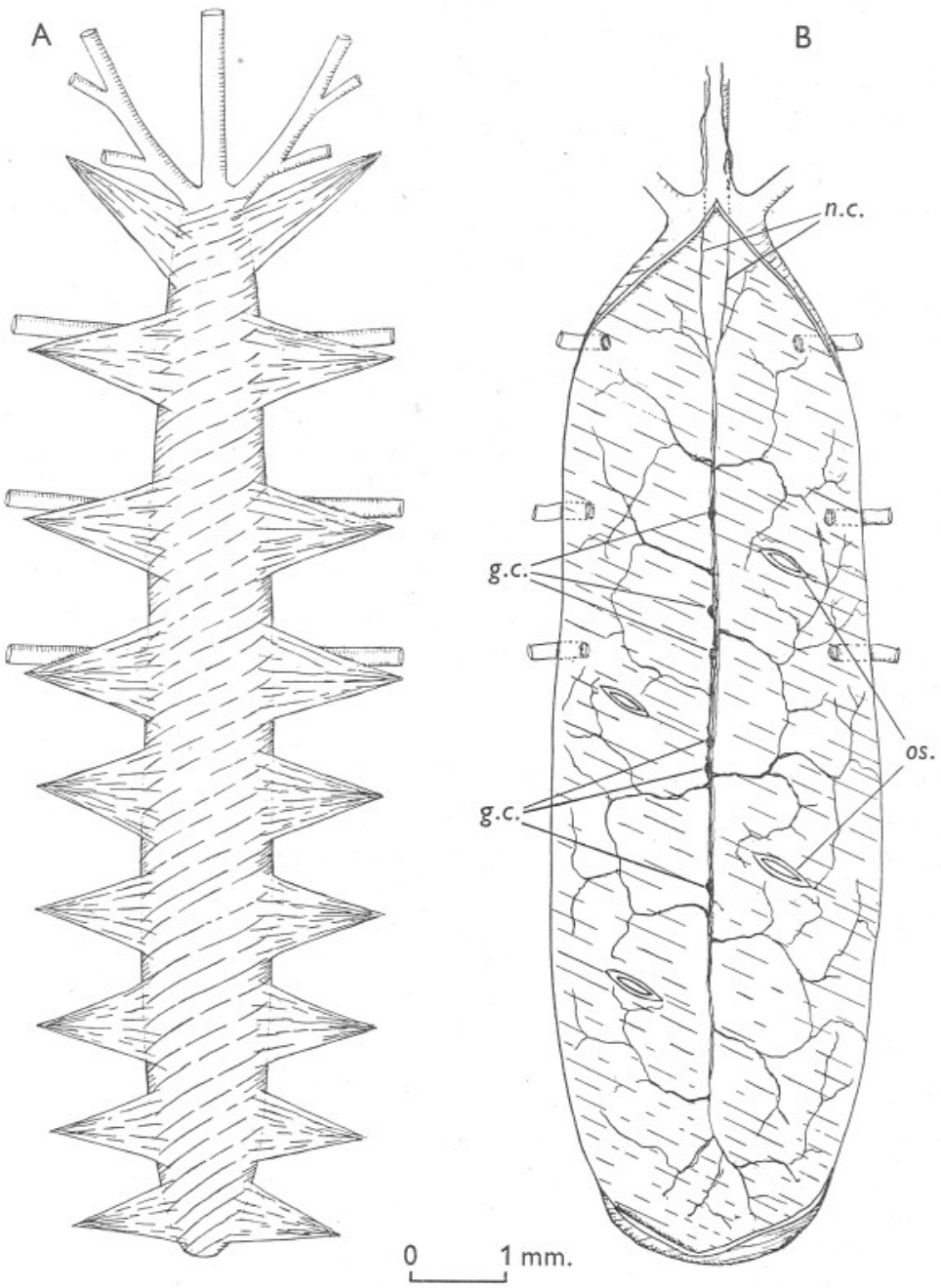

Text-fig. r. Ligia oceanica. A, view of the heart from the ventral side with its arteries and alary muscles; B, view of the heart cut in the mid-line of the ventral wall and stretched with the inner surface turned upwards. g.c., ganglion cells in the main trunk (ganglionic trunk); n.c., nerves connecting the local system with the central nervous system; os., ostia.

The ventral wall of the heart is connected with the pericardial diaphragm, a horizontal septum reinforced by muscles of triangular shape which may be called 'alary muscles' as in insects. Connective tissue fibres attached to the dorsal and lateral walls suspend the heart tube in the pericardial sinus, thus 
acting as the 'ligaments of the heart', a name given to similar elements in decapods.

As far as could be ascertained the presence of the alary muscles has not been mentioned in works on the anatomy of Isopoda, but on whole mounted preparations they can be clearly seen (P1. I, fig. I). There are nine pairs of these muscles situated as shown in Text-fig. I A, the first four a little more widely spaced from each other than the remainder. At the apex of each triangle the converging muscle fibres pass into a slender tendon attached to chitinous ridges of the anterior borders of the terga. Towards the basis of the triangle the muscle fibres pass into the connective tissue of the pericardial diaphragm.

\section{Nerves of the Heart}

As in other groups of crustaceans in which the innervation of the heart has been investigated (Alexandrowicz, I932, I934) there are in the Isopoda three systems of nerve elements connected with the heart, viz. (i) a local nervous system, (ii) nerves connecting the local system with the central nervous system, and (iii) nerves of the arterial valves (Text-fig. I B). There are, moreover, nerve elements which, though not connected directly with the heart itself, have certain relations with its function, viz. the nerves of the alary muscles and the nerves of the pericardium and the heart ligaments.

\section{Local Nervous System}

The local nervous system consists of neurons, the cell bodies of which lie in a nervous trunk situated on the inner surface of the dorsal wall and which may therefore be called the ganglionic trunk of the heart. It runs in the midline for a great part of the length of the heart tube, being thickest along the middle region and tapering towards both ends. The whole of it is shown in Text-fig. I B, and parts of it in Pl. I, figs. I-4.

\section{Ganglion Cells}

I have already given brief mention of the presence of ganglion cells in the heart of L. oceanica in my paper of I93I. In I934, S. Suzuki stated that in the heart of L. exotica six nerve cells are present, but as they were found on sections stained with non-specific methods this writer could not observe much of the innervation of the heart in this species as well as in two other isopods (Tylos granulatus and Porcellio scaber) investigated later (Suzuki, I935; Tanita, I939).

In methylene-blue preparations the nerve cells rarely stain and it is surprising to see no trace of them even in many preparations in which the nerve trunk and its branches are very well stained, although this whole system apart from a few elements of different origin is derived from the processes of these cells. They are six in all, situated at various distances from one another as shown in Text-fig. I B. In methylene-blue preparations they appear in varying 
numbers and only exceptionally are all of them seen together; therefore, in order to be certain that this number is correct I verified it on serial sections stained with ordinary methods.

Very few details of the cells can be observed, not only because they are visible merely after prolonged staining when the nerve elements begin to deteriorate, but also because of their situation in the trunk among the nerve fibres as seen in Pl. I, fig. 2. Such a picture as in Pl. I, fig. 4, showing a cell not covered by the fibres and not obscured by surrounding connective tissue, is very seldom met with. When a cell is only faintly stained a largish nucleus may be seen and fine dark blue granules in the cytoplasm. Sometimes the presence of a cell can only be recognized by these characteristic granulations. In a few cases fine nerve fibres have been observed in the vicinity of a cell, suggesting the presence of a basketwork around it, but this is much less distinct than in the ganglion cells found in other groups of crustaceans.

The processes of the cells stain badly, not only the shorter ones but also the axons at their origins. This feature, not uncommon in other nerve elements in arthropods, impedes the direct observation of the course taken by the axons belonging to each neuron of the ganglionic trunk; some evidence, however, may be gained from the composition of this trunk. It contains several fibres of stout calibre and a few thinner fibres, and it can be stated that in the middle portion of the trunk the stouter are six in number. This points to the conclusion that the axons of all the nerve cells are present here and it can also be assumed that the axons of the anterior cells are directed backwards and that those of the posterior cells run in the opposite direction.

The ganglionic trunk gives off branches which pass on to the walls of the heart. Two of the branches originating in the bifurcation of the anterior end of the trunk take a symmetrical course, diverging obliquely from one another. These are the branches which are joined by the nerves from the central nervous system. The branches arising from the trunk distribute their fibres over the whole heart as shown in Text-fig. I B and Pl. I, fig. I, and are interconnected by anastomoses. No special endings on the muscles can be seen.

It can be noticed that the branches springing from the main trunk are composed of more than one fibre each (Pl. I, fig. 4) and that these fibres originate from different elements of the trunk. It has not been possible to trace them further, but it may be inferred that in the same portion of the heart the muscles are innervated by more than one neuron of the local system and the presence of the anastomoses between the branches affords supporting evidence for this assumption.

\section{Nerves Connecting the Local System with the Central Nervous System}

The connexion of the local system with the central nervous system is established by a pair of nerves running alongside the aorta. They associate with the fibres innervating the valves of the aorta and this makes it difficult 
to trace them, but on approaching the heart they separate from other fibres and, piercing the dorsal wall of the heart near its anterior end, pass on to its inner surface (Text-fig. 2). Meeting here the two anterior branches of the ganglionic trunk they run along with them into the trunk itself. These nerves appear to be composed of more than one fibre each and at some places three

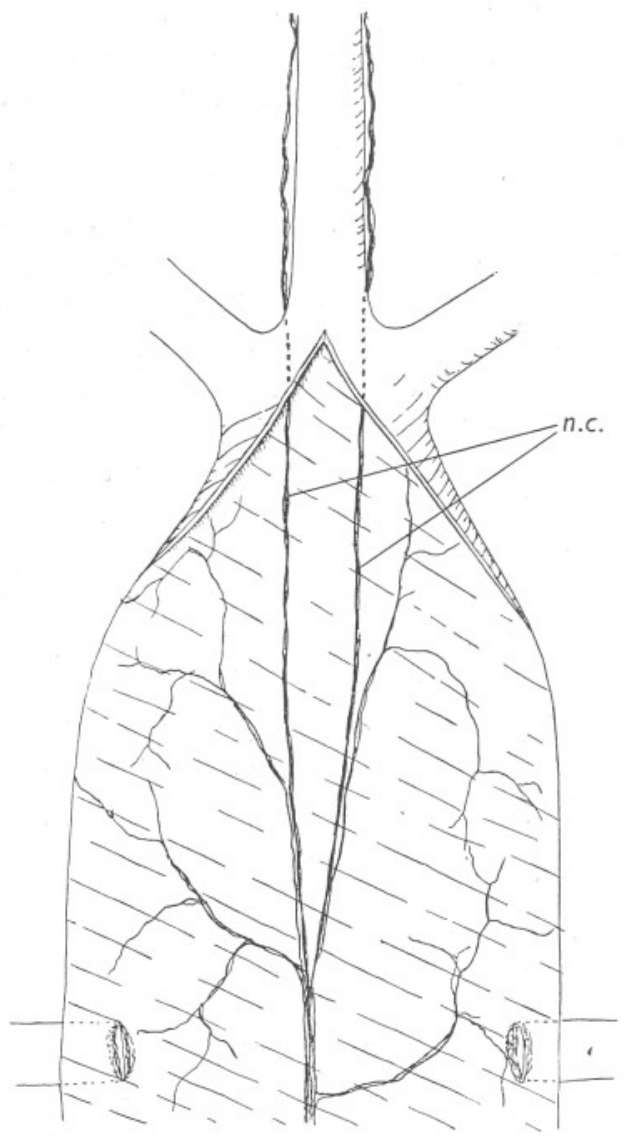

Text-fig. 2. Anterior part of the heart of Ligia oceanica from a preparation stretched as in Text-fig. I B. n.c., nerves connecting the local system with the central nervous system.

of them could be seen, but owing to the size of the nerve elements in Ligia more exact observations are difficult. The further course of these fibres in the ganglionic trunk and their connexions could not be discerned. It may be inferred only that the finer fibres seen at some places in the trunk belong to these nerves. I was also unsuccessful in my endeavours to establish the origin of these elements. As the nerves accompanying the aorta can be followed up into the first thoracic segment and even beyond its anterior border it seems probable that they arise from the suboesophageal ganglion, 
but, as I have said before, the staining of the nerves in that region has always been unsatisfactory. It is also not improbable that some fibres join these nerves during their course along the aorta, but no precise evidence could be established supporting this contention.

Suzuki believed that two pairs of nerves pass on to the dorsal wall of the heart from the abdominal ganglia and, moreover, reported the presence of small nerve cells on these fibres. I could not find such nerves in methyleneblue preparations, unless they are those which terminate on the pericardium and on the ligaments of the heart. As for the cells observed by Suzuki they were probably nerve cells of the muscle receptors, since these organs are also present in Ligia and are situated, as in decapods and stomatopods, on the dorsal muscles of the thoracic and abdominal segments at the place corresponding to that in which lie the cells seen by Suzuki.

\section{Nerves of the Arterial Valves}

All the arterial vessels arising from the heart are provided with valves the muscles of which receive their innervation from a special system of nerves.

The nerves for the valves of the aorta run down this vessel and originate somewhere in the most anterior ganglia of the central nervous system. Approaching the heart they divide into several branches, giving off numerous ramifications expanding on the valves which are apparently complicated in structure, having two main and two additional flaps.

The valves of the first pair of lateral arteries receive their innervation from two fibres. One of them runs along the anterior wall of this vessel having an antero-lateral course, the other having a more transverse direction and, near the valve, lying close to the posterior wall of the artery (Text-fig. 3A). Each of them associates more proximally with branches of motor nerves. The nerves for the valves of the following three pairs of arteries run from the ventral ganglionic cord with the motor fibres supplying the dorsal muscles of their respective segments. Owing to the different appearance of the nerves of the valves it may be assumed that they are of some special kind and that, apart from taking the same route as the motor fibres, they have nothing in common with the latter.

The distribution of the nerve fibres in the valves gives a characteristic picture of very abundant, densely arranged, tortuous ramifications. They are confined to the valves and when even nothing but the nerves are stained the outlines of the valves are well defined by their endings.

\section{Nerve of the Alary Muscles}

The nerve supplying the alary muscles runs from the central nervous system with the motor nerve of the dorsal muscles and, although its proximal course and its origin could not be traced, it may be assumed as for the nerves of the valves that it does not belong to the same category as the motor nerves 
of the ordinary muscles. It comes near the heart behind the fourth (last) pair of arteries and bifurcates here into branches running in opposite directions (Text-fig. 3 B). They have a characteristic sinuous course passing across the

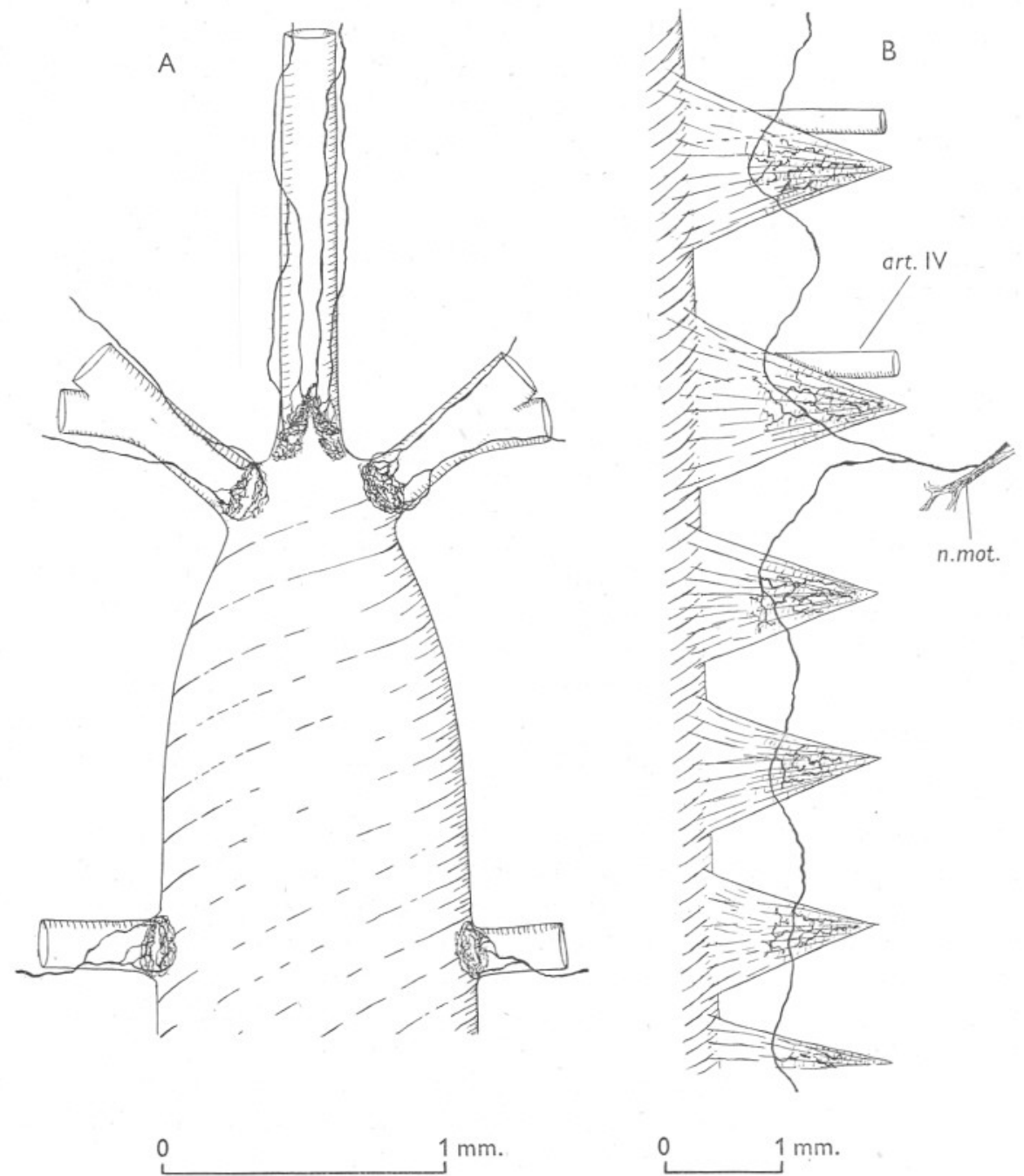

Text-fig. 3. A, anterior part of the heart of Ligia oceanica, showing the nerves of the valves of the aorta and of the two first pairs of arteries; $\mathrm{B}$, innervation of the alary muscles. n.mot., motor nerve from which the nerve of the alary muscles is separating; art. IV, artery of the fourth pair.

dorsal sides of the alary muscles and sending to them short fibres ramifying in a pattern differing from that seen in ordinary muscles. The posterior branch is easily followed in its course to all posterior alary muscles. As to the anterior 
branch I am not quite certain whether it supplies all the four anterior muscles. This uncertainty is to a great extent due to the presence in this region of the nerves of the arterial valves which complicate the picture. I tried to find out if the innervation of the valves is not in some way linked with that of the alary muscles, but no convincing evidence of such a connexion could be obtained.

\section{COMPARISON OF THE INNERVATION OF THE HEART IN ISOPODA WITH THAT IN OTHER CRUSTACEA}

On comparison of the innervation of the heart of isopods with that of the two groups of crustaceans in which it is better known, i.e. in decapods and stomatopods, it becomes obvious that in all these animals it is built up on the same lines: there is everywhere a local nervous system consisting of neurons the cell bodies of which are situated in the heart itself and the processes of which spread over all its muscle fibres. The number of these neurons (six) in Ligia oceanica is smaller than in other species investigated (sixteen in Astacus fluviatilis, nine in other Macrura, in Anomura and Brachyura, sixteen in Stomatopoda). The nerves connecting the local neurons with the central nervous system show fewer details in Ligia than in the other Crustacea. In the latter these nerves, called 'dorsal heart nerves', have been found to contain two kinds of fibres one of which is represented by three thicker elements. As mentioned before, it was neither possible to state whether in Ligia they are of two sorts, nor what might be their number.

The innervation of the arterial valves has much in common in all these crustaceans, being in all of them independent of the local system, and in all animals the valves of the paired arteries have a segmental innervation, whereas the valves of the anterior aorta receive their supply from a nerve or nerves running along this vessel.

This nerve running down the aorta was the first to be discovered to have a relation with the heart in crustaceans. As it had been known for many years by the name of nervus cardiacus, I left this term when describing the innervation of the heart in decapods, adding only the word 'anterior' although it proved to end in the valve and not to pass farther into the heart. It may now seem inconsistent not to use the same term for the homologous elements in the Isopoda. The reason is that in the latter there are other nerves following the same route and which deserve even more the name 'anterior nerves' of the heart since they pass into its ganglionic trunk. They are most probably homologous with those nerves which in decapods and stomatopods were described as nervi cardiaci dorsales, but again, because of their topography, the latter term does not fit them. As for the term 'regulator nerves', also suggested by me previously, it may be objected that it is not advisable to derive a name of an organ from a function which is only probable. There is an obvious need of a unified nomenclature and for the adoption of terms which could be applied generally, taking into account the fact that homologous elements may have different topographical arrangements in different animals. 
In both the Isopoda and the Stomatopoda the nerves of the arteries in any one segment are independent of those of the neighbouring segments. In Ligia even the valves of the same pair of arteries have completely separate nerves, whilst in Squilla they are connected by anastomoses.

The presence of the alary muscles both in isopods and stomatopods and their obviously similar function furthering the circulation in the big blood sinuses would suggest that there should be common features in their nerve supply. As to stomatopods, it has been stated that their alary muscles are innervated by branches belonging to the system of median connectives generally called 'unpaired nerve' (Alexandrowicz, I952). Such median connectives are present in Isopoda too, but as yet I have been unable to find out their peripheral expansion and to state whether the origin of the nerves of the alary muscles can be traced to them. This problem, as well as that of the innervation of the pericardium and the heart ligaments, might perhaps be solved by further investigations.

As regards the function of the nerve elements in the heart of Ligia it could be shown in the experiments previously recorded that the action of the heart is ruled by the local nervous system, since by severing this trunk different rhythms of pulsations were produced in different parts of the heart. As to the nerves connecting the heart with the central nervous system, it may be supposed that they have a regulating function carrying accelerating and inhibiting impulses.

\section{SUMMARY}

In the heart of Ligia oceanica (Crustacea, Isopoda) three systems of nerve elements have been distinguished: (i) a local system, (ii) nerves connecting the local system with the central nervous system, and (iii) nerves of the arterial valves.

The local nervous system of the heart is made up of six neurons which form a ganglionic trunk situated on the inner surface of the dorsal heart wall. The branches arising from this trunk expand over all the muscle fibres of the heart.

The nerves connecting the heart with the central nervous system run down the aorta and, piercing the heart wall, join the ganglionic trunk. The same route along the aorta is taken by the nerves of its valves. The valves of the four paired arteries are supplied by segmental nerves. The nerves of arterial valves have no connexion with the local nervous system of the heart.

It has been found that the pericardial diaphragm is strengthened by nine pairs of triangular 'alary' muscles. They have a particular innervation of their own, belonging neither to any of the systems of heart nerves nor to the category of motor nerves of the ordinary muscles. 


\section{REFERENCES}

Alexandrowicz, J. S., I93I. Quelques expériences sur le fonctionnement du système nerveux du cœur des Crustacés Isopodes. C.R. Soc. Biol., Paris, T. I08, pp. I270-2.

1932. The innervation of the heart of the Crustacea. I. Decapoda. Quart. Fourn. Micr. Sci., Vol. 75, pp. 18I-249.

- 1934. The innervation of the heart of the Crustacea. II. Stomatopoda. Quart. fourn. Micr. Sci., Vol. 76, pp. 51 I-48.

- 195I. Muscle receptor organs in the abdomen of Homarus vulgaris and Palinurus vulgaris. Quart. Fourn. Micr. Sci., Vol. 92, pp. 163-99.

- 1952. Notes on the nervous system in the Stomatopoda. I. The system of median connectives. Publ. Staz. Zool. Napoli, Vol. 23.

Delage, Y., I88I. Contribution à l'étude de l'appareil circulatoire des Crustacés Édriophthalmes marins. Arch. Zool. Exp. Gén., T. 9, pp. I-I72.

Gordon HewitT, C., I907. Ligia. L.M.B.C. Memoirs.

Suzuki, S. I934. Ganglion cells in the heart of Ligia exotica (Roux). Sci. Rep. Tohoku Imp. Univ., Vol. 9, pp. 213-17.

- 1935. On the presence of ganglion cells in some crustacean hearts. Sci. Rep. Tohoku Imp. Univ., Vol. Io, pp. 4I7-26.

ГANITA (SuzukI), S. I939. Note on the presence of ganglion cells in the heart of Porcellio scaber Latreille. Sci. Rep. Tohoku Imp. Univ., Vol. I4, pp. 21-3. 


\section{EXPLANATION OF PLATE I}

All photomicrographs have been made from preparations of the heart of Ligia oceanica stained with methylene blue, fixed in ammonium molybdate and mounted in xylol dammar. The heart wall is shown from its inner surface.

Fig. I. Part of the heart with the ganglionic trunk and its branches. Two alary muscles are seen on the left side. The dark spots on the left side of the figure are the pigmented cells.

Fig. 2. The ganglionic trunk and strands of connective tissue elements. g.c., ganglion cell.

Fig. 3. The ganglionic trunk and the muscle layer of the heart with an ostium. The fibres crossing the ostial orifice belong to one of the ligaments of the heart and are situated on the exterior side of the heart wall.

Fig. 4. Nerve cell of the ganglionic trunk. The nerve branching from the trunk has two roots proving its composition of two fibres. 


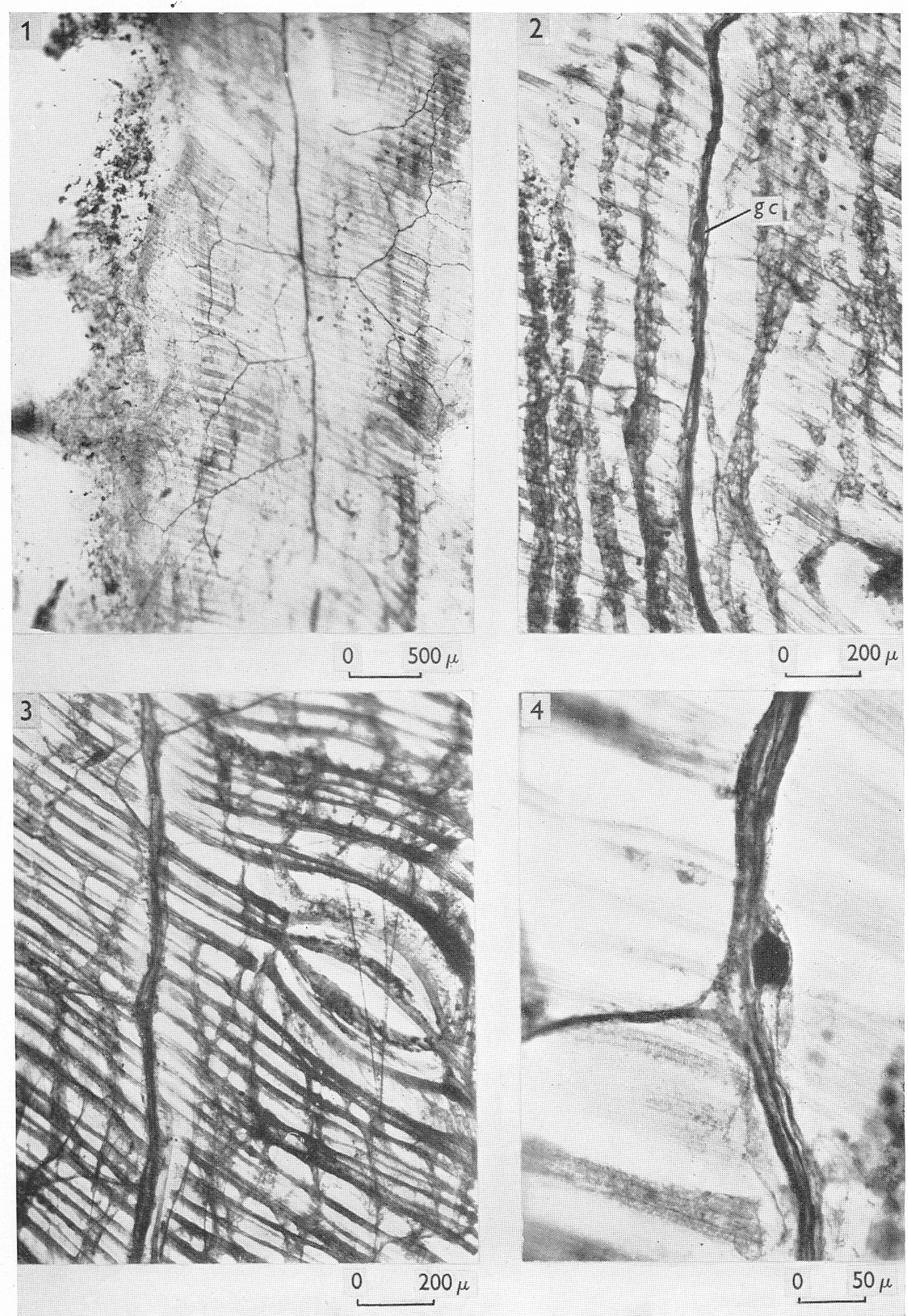

